\title{
Lipid and oxylipin profiles during aging and sprout development in potato tubers (Solanum tuberosum L.)
}

\author{
Marie-Laure Fauconnier ${ }^{\mathrm{a}, *}$, Ruth Welti ${ }^{\mathrm{b}}$, Elizabeth Blée ${ }^{\mathrm{c}}$, Michel Marlier ${ }^{\mathrm{a}}$ \\ ${ }^{a}$ Unité de Chimie Générale et Organique, Faculté Universitaire des Sciences Agronomiques de Gembloux, Passage des Déportés 2, \\ B-5030 Gembloux, Belgium \\ ${ }^{\mathrm{b}}$ Division of Biology, Ackert Hall, Kansas State University, Manhattan, KS 66506, USA \\ ${ }^{\mathrm{c}}$ Laboratoire des Phytooxylipines, IBMP-CNRS-UPR 2357, Rue Goethe 28, 67083 Strasbourg Cedex, France
}

Received 18 February 2003; received in revised form 5 June 2003; accepted 19 June 2003

\begin{abstract}
Potato tubers (Solanum tuberosum L. cv Bintje) were stored at $20{ }^{\circ} \mathrm{C}$ for 210 days without desprouting to study the lipoxygenase pathway during aging. After 15 days of storage, potato tubers sprouted, while after 45-60 days, apical dominance was lost and multiple sprouts developed. Analysis of the fatty acid hydroperoxides (HPOs) revealed that 9-S-hydroperoxide of linoleic acid (9-HPOD) was the main oxylipin formed. Between 45 and 60 days of storage, increases in the levels of 9-HPOD and colneleic acid were observed. Analysis of phospholipids and galactolipids by electrospray ionisation tandem mass spectrometry (ESI-MS/MS) showed that a decrease in the levels of phosphatidylcholine (PC), phosphatidylethanolamine (PE), phosphatidylinositol (PI), digalactosyldiacylglycerol (DGDG), and monogalactosyldiacylglycerol (MGDG) occurred between 0 and 45 days of aging. The decrease in the amount of linoleic acid in complex lipids correlates well with the amount of 9-HPOD and colneleic acid produced.
\end{abstract}

(C) 2003 Elsevier B.V. All rights reserved.

Keywords: Oxylipin; Phospholipid; Lipoxygenase pathway; Solanum tuberosum L.; Potato tuber; Colneleic acid

\section{Introduction}

Fatty acid hydroperoxides (HPOs) are formed by autoxidation or by the action of enzymes such as lipoxygenase (LOX) or $\alpha$-dioxygenase on polyunsaturated fatty acids [1]. HPOs are key intermediates in the LOX pathway; they can be converted enzymatically to a variety of compounds called oxylipins. At least seven enzymatic pathways use HPOs as substrates: hydroperoxide lyase that cleaves HPO

\footnotetext{
Abbreviations: DBI, double bond index; DES, divinyl ether synthase; DGDG, digalactosyldiacylglycerol; ESI-MS/MS, electrospray ionisation tandem mass spectrometry; GC-MS, gas chromatography-mass spectrometry; 9-HPOD, 9-hydroperoxide of linoleic acid; 9-HPOT, 9-hydroperoxide of linolenic acid; 13-HPOD, 13 hydroperoxide of linoleic acid; 13-HPOT, 13-hydroperoxide of linolenic acid; HPO, fatty acid hydroperoxide; LOX, lipoxygenase; LysoPC, lysophosphatidylcholine; LysoPE, lysophosphatidylethanolamine; LysoPG, lysophosphatidylglycerol; MGDG, monogalactosyldiacylglycerol; PA, phosphatidic acid; PC, phosphatidylcholine; PE, phosphatidylethanolamine; PG, phosphatidylglycerol; PI, phosphatidylinositol

* Corresponding author. Tel.: +32-81-622292; fax: +32-81-622227.

E-mail address: fauconnier.ml@fsagx.ac.be (M.-L. Fauconnier).
}

into aldehydes and oxo-acids, allene oxide synthase that forms precursors of $\alpha$ - and $\gamma$-ketols and jasmonic acid, peroxygenase that causes epoxidation and reduction of HPO, epoxy alcohol synthase that transforms HPO into epoxy hydroxy fatty acids, the reductase pathway that reduces HPO into hydroxyderivatives, divinyl ether synthase (DES) that produces divinyl ethers, and finally, LOX that acts on HPO to give keto derivatives [2].

Colneleic acid and colnelenic acid are oxylipins formed by the action of DES on the 9-hydroperoxide of linoleic acid (9-HPOD) and the 9-hydroperoxide of linolenic acid (9HPOT), respectively [3]. The DES pathway was discovered in potato tubers by Galliard and Phillips in 1972 [4]. Afterwards, colneleic and colnelenic acids were identified in tomato roots [5] and potato leaves [6]. The 13-hydroperoxides of linoleic (13-HPOD) and linolenic (13-HPOT) acid can also serve as substrates for DES, resulting in etheroleic and etherolenic acid in garlic bulbs [7] or $R a$ nunculus plants [8,9]. DES activity has also been demonstrated in algae $[10,11]$.

Oxylipins are believed to play an important role in plant defence against pathogens and in response to stress con- 
ditions such as injury $[1,12]$. Volatile oxylipins have also been shown to act as signaling molecules between plants [13]. While there has been much study of jasmonic acid in the past decade, recently the importance of other oxylipin pathways in plant resistance to pathogens has been revealed. In potato plants, Weber et al. [6] showed that the divinyl ethers colneleic and colnelenic acids had an inhibitory effect on Phytophthora infestans, the causal agent of late blight disease, suggesting a function in plant defence for DES. In potato cell suspension cultures treated with elicitor from $P$. infestans, the DES pathway was the major pathway induced [14-16].

Although our knowledge of oxylipins' role in the defence of plants against pathogens and stress is increasing rapidly, almost nothing is known about the role of these molecules in aging and senescence. Even though LOX is often proposed to be responsible for peroxidative damage to membrane lipids during aging and senescence, its role is far from clear. LOX activity increases during senescence of rose petals [17], daylily petals [18], carnation petals [19], and pea foliage [20], but clearly decreases during soybean seed aging [21], soybean cotyledon senescence [22] or in detached wheat and rye leaves [23]. In a previous study [24], we showed that in potato tubers stored at $20{ }^{\circ} \mathrm{C}$, LOX activity and the total HPO content decreased during aging, even during the period after 120 days when membrane integrity dropped drastically. The few previous studies on the LOX pathway during aging or senescence have determined only total LOX activity; in this study, we have characterised in detail the fatty acid HPOs and their products that accumulated during tuber storage. We have also investigated the origin of the precursors of HPO, i.e. the polyunsaturated fatty acids that are potentially derived from phospholipids and galactolipids.

\section{Materials and methods}

\subsection{Plant material and sampling}

Potato tubers (Solanum tuberosum L. cv Bintje) were obtained at harvest from a local market. Tubers with lengths between 60 and $65 \mathrm{~mm}$ were selected for the study. The selected tubers were washed and stored for 2 weeks at room temperature to allow wound healing. The ages were calculated from the end of the wound-healing period. The tubers were stored for 210 days at $20{ }^{\circ} \mathrm{C}$ with a relative humidity of $90 \%$ in the dark without desprouting. Samples were taken after $0,15,30,45,60$, $90,120,150,180$ and 210 days. For each sampling, 10 tubers were randomly taken and cut into two parts transversely; sprouts, when present, were removed before sampling. In each tuber half, a core was taken with a punch $(18 \mathrm{~mm}$ in diameter) perpendicular to the cutting axis. The cylinders were then cut, and material from the centre of the tuber was discarded, so that the remaining core weighed $10 \mathrm{~g}$. The samples were immediately frozen in liquid nitrogen, powdered in a mill and stored at $-80{ }^{\circ} \mathrm{C}$. The last operations were performed rapidly to minimize wounding responses during sampling. Analysis of a sample that had not been stored at $20{ }^{\circ} \mathrm{C}$, after 0,6 , and 12 months at $-80{ }^{\circ} \mathrm{C}$ indicated that storage at $-80{ }^{\circ} \mathrm{C}$ did not result in autoxidation of lipids.

\subsection{Phospholipid and galactolipid analysis}

\subsubsection{Extraction}

Two milliliters of isopropanol $(0.01 \% \mathrm{w} / \mathrm{v}$ BHT $)$ at $75{ }^{\circ} \mathrm{C}$ were added to $1 \mathrm{~g}$ of potato powder. The mixture was incubated at $75{ }^{\circ} \mathrm{C}$ for $15 \mathrm{~min}$, followed by addition of 1 $\mathrm{ml}$ of chloroform and $0.8 \mathrm{ml}$ of distilled water. The tubes were shaken for $30 \mathrm{~min}$ at room temperature and centrifuged $10 \mathrm{~min}$ at $1200 \times \mathrm{g}$. The liquid was removed and the plant material was re-extracted with chloroform/methanol (2:1) containing $0.01 \% \mathrm{w} / \mathrm{v}$ BHT for $30 \mathrm{~min}$. This last operation was repeated five times and the extraction liquids were combined, washed with $2 \mathrm{ml}$ of $1 \mathrm{M} \mathrm{KCl}$, filtered, and evaporated to dryness under nitrogen. The lipid extract was redissolved in $1.5 \mathrm{ml}$ of chloroform $/$ methanol $(2: 1, \mathrm{v} / \mathrm{v})$. The lipid extracts were stored at $-80{ }^{\circ} \mathrm{C}$ until analysis.

\subsubsection{Analysis}

Phospholipid and galactolipid analyses were performed by electrospray ionisation tandem mass spectrometry (ESIMS/MS) using internal standards as described in [25], except that, for phospholipid analysis, $150 \mu \mathrm{l}$ of extract were used, and for galactolipid analysis, $225 \mu \mathrm{l}$ of extract were used. The extraction and analysis were performed on five independent samples of potato tubers at each time point. Each lipid species amount shown is the mean of the five determinations + standard deviation.

\subsection{Quantitation of 9- and 13-HPOD, HPOT, colneleic} acid, and colnelenic acid by HPLC

\subsubsection{Extraction}

Four hundred microliters of $1.8 \times 10^{-4} \mathrm{M} 15$-HEDE (15-hydroxy-11,13 eicosadienoic acid, internal standard) were added to $5 \mathrm{~g}$ of potato tuber powder that was extracted with $15 \mathrm{ml}$ of diethyl ether at room temperature for $10 \mathrm{~min}$. The powder was re-extracted with $10 \mathrm{ml}$ of diethyl ether for $10 \mathrm{~min}$ at room temperature and the ether fractions were pooled and dried with anhydrous sodium sulfate. Extraction yields, which were determined by addition of a known amount of each compound to potato powder before analysis, were $87 \pm 3 \%(n=5)$ for 15 HEDE (the internal standard), $85 \pm 2.5 \%(n=5)$ for 13 HPOT, and $82 \pm 4 \%(n=5)$ for colneleic acid. The solvent was evaporated to dryness under nitrogen and the extracted compounds were redissolved in $100 \mu \mathrm{l}$ of acetonitrile. A 
blank was made with the same protocol omitting the potato powder.

\subsubsection{Analysis}

The analysis was performed with a Hewlett Packard series 1050 HPLC (with a diode array detector) on an Inertsil $250 \times 4.6 \mathrm{~mm} 5$ ODS-2 column (Chrompack, Middelburg, The Netherlands) with a flow rate of 0.7 $\mathrm{ml} / \mathrm{min}$. Solvent A was water containing $0.01 \% \mathrm{v} / \mathrm{v}$ trifluoroacetic acid; solvent $\mathrm{B}$ was acetonitrile containing $0.01 \% \mathrm{v} / \mathrm{v}$ trifluoroacetic acid. Elution conditions were: 0-61 min, 47\% solvent $\mathrm{A} ; 61-63 \mathrm{~min}$, a gradient from $47 \%$ A to $20 \% \mathrm{~A} ; 63-74 \mathrm{~min}, 20 \% \mathrm{~A} ; 74-75 \mathrm{~min}$, from $20 \%$ A to $0 \% \mathrm{~A} ; 75-95 \mathrm{~min}, 100 \% \mathrm{~B}$. For re-equilibration, the flow was continued from 95 to $100 \mathrm{~min}$ with a gradient from $0 \% \mathrm{~A}$ to $47 \% \mathrm{~A}$ and from 100 to $105 \mathrm{~min}$ at $47 \%$ A. Identification of 9- and 13-HPOD and HPOT and of colneleic and colneleic acid was based on their UV spectra and retention time by comparison with pure compounds synthesised, extracted, purified by preparative HPLC, derivatised, and characterised by gas chromatography-mass spectrometry (GC-MS) analysis according to Fauconnier et al. [26] for HPO and to Weber et al. [6] for colneleic and colnelenic acid. All the experiments were performed in triplicate (on three independent potato tuber extracts) and the results shown are the means \pm standard deviations.

\subsection{Quantitation of HPO formed by autoxidation}

\subsubsection{Extraction}

Four hundred microliters of $1.8 \times 10^{-4} \mathrm{M}$ 15-HEDE (15-hydroxy-11,13-eicosadienoic acid, internal standard) were added to $1 \mathrm{~g}$ of potato tuber powder which was homogenised with $2 \mathrm{ml}$ of $0.2 \mathrm{~N} \mathrm{NaOH}$ containing $5 \%$ $(\mathrm{w} / \mathrm{v})$ of $\mathrm{NaBH}_{4}$ at $4{ }^{\circ} \mathrm{C}$ for $45 \mathrm{~s}$ with a Waring blender. The mixture was acidified with $60 \% \mathrm{HClO}_{4}(\mathrm{v} / \mathrm{v})$ to $\mathrm{pH} 4$ and extracted with $5 \mathrm{ml}$ of methanol/chloroform $(50: 50, \mathrm{v} / \mathrm{v})$. The mixture was centrifuged $5 \mathrm{~min}$ at $1200 \times g$ to allow phase separation; the chloroform phase was removed and the upper phase was re-extracted twice with $4 \mathrm{ml}$ of chloroform. The chloroform phases were pooled and evaporated to dryness with a rotary evaporator at room temperature. The extract was redissolved in $1.5 \mathrm{ml}$ of hexane/ diethyl ether $(70: 30, \mathrm{v} / \mathrm{v})$. It can be noted that preformed hydroxy acids, present in very low amounts in potato, were included in the determination of fatty acid HPOs, when this reduction method was utilized.

\subsubsection{Analysis}

Non-enzymatic formation of oxylipins was assessed by comparing the amounts of species that are formed both enzymatically and non-enzymatically with the amount of species that are formed only non-enzymatically. HPO were quantified as their hydroxyderivatives by HPLC using standards to identify HPO isomers [27].

\subsection{Chiral analysis of HPOs}

\subsubsection{Extraction}

The extraction was performed as described in Section 2.3 , but using $25 \mathrm{~g}$ of potato tubers and redissolving the extracts in $3 \mathrm{ml}$ of acetonitrile.

\subsubsection{Purification of 9- and 13-HPOD by preparative HPLC}

The purification was performed on a Hewlett Packard HP 1050 HPLC using a diode array detector with detection at $234 \mathrm{~nm}$. The separation was performed on an Inertsil 5 ODS-2 $(250 \times 10 \mathrm{~mm})$ column. Five hundred microliters were injected, and the flow rate was $2.6 \mathrm{ml} / \mathrm{min}$. Solvent A was water containing trifluoroacetic acid at $0.01 \% \mathrm{v} / \mathrm{v}$, solvent B was acetonitrile containing $0.01 \% \mathrm{v} / \mathrm{v}$ trifluoroacetic acid. The solvent sequence for elution was: $0-4 \mathrm{~min}$, $50 \%$ A to $40 \% \mathrm{~A} ; 4-30 \mathrm{~min}, 40 \% \mathrm{~A} ; 30-32 \mathrm{~min}, 40-35 \%$ A; 32-39 min 35\% A; 39-40 min, 35-0\% A; 40-55 min, $100 \% \mathrm{~B}$. For re-equilibration, the flow was continued from 55 to 60 min with a gradient from $0 \%$ to $50 \% \mathrm{~A}$, and from 60 to $65 \mathrm{~min}$ at $50 \% \mathrm{~A}$. The fractions were collected based on their retention time and UV spectra. Solvent was evaporated to dryness, and the samples were redissolved in $1 \mathrm{ml}$ of diethyl ether and reduced by addition of $2 \mathrm{mg}$ of $\mathrm{NaBH}_{4}$. After 10 min of reaction at $4{ }^{\circ} \mathrm{C}$, the ether fraction was removed, and the solvent was evaporated to dryness under nitrogen. The samples were redissolved in $1 \mathrm{ml}$ of methanol.

\subsubsection{Chiral analysis of hydroxyderivatives}

The analysis was performed with a Chiralcel OD column $(250 \times 4.6 \mathrm{~mm}$ from Diacel Chemical Industries, Interchim, France) [28].

\subsection{GC-MS analysis of oxylipins}

\subsubsection{Extraction and esterification}

Potato tuber powder $(25 \mathrm{~g})$ was extracted with $75 \mathrm{ml}$ of diethyl ether at room temperature for $10 \mathrm{~min}$. The organic phase was removed and the potato powder was extracted again with $75 \mathrm{ml}$ of diethyl ether. The ether extracts were pooled, filtered and dried with anhydrous sodium sulfate. The solvent was evaporated to dryness with a rotary evaporator and redissolved in $1.5 \mathrm{ml}$ of diethyl ether. The extract was esterified with $2 \mathrm{ml}$ of diazomethane for $10 \mathrm{~min}$ at $0{ }^{\circ} \mathrm{C}$.

\subsubsection{Analysis}

Analysis was performed on an Agilent Technologies 6890 GC-MS with a Agilent Technologies HP 5 MS column $(30 \mathrm{~m} \times 0.25 \mathrm{~mm}$ with a phase thickness of $0.25 \mu \mathrm{m})$. The splitless injector was at $275{ }^{\circ} \mathrm{C}$, and the temperature programme was: $1 \mathrm{~min}$ at $35{ }^{\circ} \mathrm{C}$, then from 35 to $300{ }^{\circ} \mathrm{C}$ at $5{ }^{\circ} \mathrm{C}$ per minute. The ionisation potential was fixed at 70 $\mathrm{eV}$ with a mass range of 50-550 a.m.u. The injected volume was $1 \mu \mathrm{l}$. The acquisition was also performed in 
the Selective Ion Monitoring (SIM) mode by choosing three characteristic ions for each oxylipin of interest.

\section{Results and discussion}

\subsection{Physiological changes during potato tuber aging}

Potato tubers are organs of vegetative propagation that can maintain viability up to 3 years under optimal storage conditions: low temperature and high relative humidity. Under our experimental conditions $\left(20^{\circ} \mathrm{C}\right)$, potato tubers sprout rapidly and undergo accelerated aging. After 210 days of storage, the fresh weight of the tubers decreased $22 \pm 2 \%$ (average of 10 determinations). During storage of potato tubers, four stages of physiological development could be described [29]: (1) dormancy, during which there is no sprouting even under favourable conditions, which corresponded to the period from 0 to 15 days under our conditions, (2) apical dominance, in which only one sprout develops, which corresponded to the period from 15 to 45 days, (3) the multiple sprout stage, in which apical dominance is released and more than one bud sprouts at the same time, which began around 45-60 days, and (4) the daughter tuber stage, in which sprouts are replaced by daughter tubers appearing directly on the mother tuber; this stage did not occur during the 210 days of aging in our experiments.

\subsection{HPOs during aging of potato tubers}

The extraction and HPLC method that we have developed allow the rapid and single-step determination of individual HPOs, colneleic and colnelenic acid. As we previously described [30], because potato tubers don't contain large amount of HPO lyase, HPOs can be extracted and quantified without reduction to the corresponding

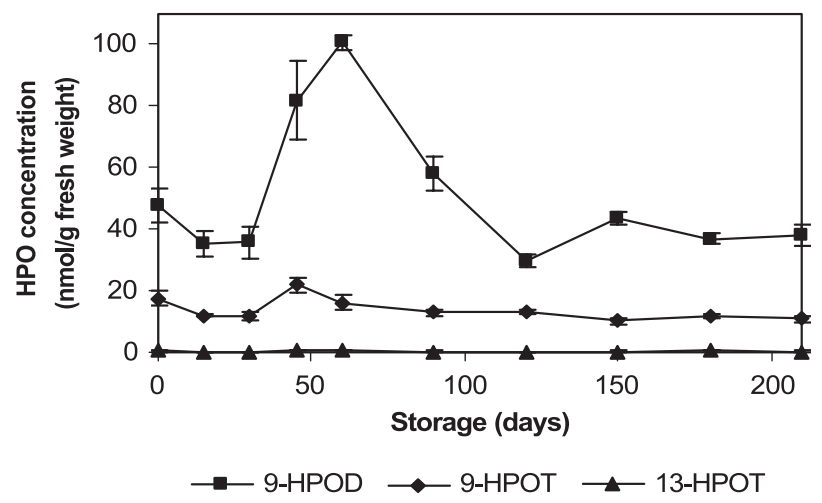

Fig. 1. Fatty acid hydroperoxide concentrations during aging of potato tubers. HPO were extracted by diethyl ether without reduction and analysed by HPLC. The analysis was performed on three independent extracts. 9-HPOD, 9-hydroperoxide of linoleic acid; 9-HPOT, 9-hydroperoxide of linolenic acid; 13-HPOD, 13 hydroperoxide of linoleic acid; 13-HPOT, 13-hydroperoxide of linolenic acid.
Table 1

Percentage of 9-, 12-, 13- and 16-HPOD and HPOT formed during storage of potato tuber (percentage of the total HPOD and HPOT amounts)

\begin{tabular}{llll}
\hline $\begin{array}{l}\text { Storage } \\
\text { time } \\
\text { (days) }\end{array}$ & $\begin{array}{l}\text { 9-HPOD and } \\
\text { 9-HPOT }\end{array}$ & $\begin{array}{l}\text { 13-HPOD and } \text { otal }) \\
\text { 13-HPOT } \\
\text { (\% of total) }\end{array}$ & $\begin{array}{l}\text { 12-HPOT and } \\
\text { (6-HPOT of total) }\end{array}$ \\
\hline 0 & 84.9 & 12.7 & 2.4 \\
60 & 81.8 & 15.1 & 3.1 \\
210 & 69.1 & 22.4 & 8.5 \\
\hline
\end{tabular}

The analysis was performed by HPLC after reduction of the lipid hydroperoxides to their corresponding alcohols.

alcohols. Omitting the reduction step is more rapid and allows separate determination of hydroxy fatty acids that may be formed in the plant. Hydroxy fatty acids were identified in the samples on the basis of their retention time and UV spectrum, compared to standards, but the hydroxy fatty acid concentration was so low that the quantification was not possible. The HPO concentration during storage is presented in Fig. 1 (nmol/g fresh weight). Calculation of these quantities took extraction yield into account, as hydroperoxides, represented by 13-HPOT, and 15-HEDE (the internal standard) were determined to have similar extraction yields: $85 \pm 3 \%(n=5)$ for 13 -HPOT and $87 \pm 3 \%(n=5)$ for 15 -HEDE. The 9-isomers of HPO, in particular 9-HPOD, were the main species formed in potato tubers. The formation of higher levels of HPOD than HPOT is consistent with the higher levels of HPOD's precursor, 18:2, than HPOT's precursor, 18:3, in potato tubers [24]. 13-HPOD was present in the tuber at very low concentration, but its levels are not shown in the figure, as it is difficult to quantify because its retention time is very close to that of 9-HPOD. The maximal concentration of 9HPOT was reached at 45 days, and the maximal concentration of 9-HPOD was reached at 60 days. Following these peaks, the HPO concentration dropped and remained low for all the isomers until the end of the experiment (210 days).

To determine whether the origins of the HPOs were enzymatic, analysis of autoxidation products and chiral analysis of the HPOs were performed. Table 1 shows the percentage of 12- and 16-HPOT formed by autoxidation compared to the 9- and 13-HPOD and HPOT that can be either formed enzymatically or by autoxidation. The low levels of 12- and 16-HPOT indicated that autoxidation was only responsible for a small part of the HPO present in the tuber. During aging, the autoxidation of fatty acids increased, but the phenomenon was still quite limited. In senescing leaf, non-enzymatic lipid peroxidation has been demonstrated to play a dominant role in lipid peroxidation [31], but this was clearly not the case in potato tubers even after 210 days of storage at $20{ }^{\circ} \mathrm{C}$. Chiral analysis of 9and 13-HPOD after HPLC purification of the sample from 0 days of storage confirmed the importance of the enzymatic formation of these species. There was a large enantiomeric excess of the $S$ form; $67 \%$ of 13-HPOD was in the $S$ form, and $91 \%$ of the 9 -HPOD was in the 
$S$ form. Thus, it is apparent that lipoxygenase and, in particular, LOX-1 was mainly responsible for the synthesis of HPO in the tubers. Indeed, the 9-isomers of HPOs were the major HPOs formed, consistent with the specificity of LOX-1 [32].

\subsection{Oxylipins during aging of potato tubers}

HPLC analysis of a crude diethyl ether extract of potato tuber revealed only the presence of HPOs, colneleic acid, colnelenic acid, and slight traces of hydroxy fatty acids, whatever the storage time or the wavelength of the UV detector (diode array). Colneleic and colnelenic acid content in the tuber during aging are shown in Fig. 2 (nmol/g fresh weight). Again, the measured quantities took into account extraction yield, as the measured extraction yields of colneleic acid $(82 \pm 4 \%, n=5)$ and of the internal standard, 15HEDE $(87 \pm 3 \%, n=5)$, were similar. Colneleic acid was present in larger quantities than colnelenic acid, which is in agreement with the higher concentration of 9-HPOD, the precursor of colneleic acid, compared to 9-HPOT, the precursor of colnelenic acid. The time course of the changes in colneleic acid levels during the storage was quite similar to that of the changes in 9-HPOD levels. Although the data show that at the initial time point, colneleic acid levels are high and HPOD levels are low, our previous work has shown that tuber metabolism at the start of storage can be variable, probably due to postharvest stress [24]. While initial metabolism may be erratic, changes later in the aging process are reproducible. Both the colneleic acid and 9HPOD levels showed a clear increase around 45 days of storage, but colneleic acid was always five to ten times less concentrated than 9-HPOD. The phenomenon could be explained by two possible mechanisms: 9-HPOD was converted by other enzymatic systems to other oxylipins, or colneleic acid was the main species formed but it was converted to other species.

To identify other oxylipins potentially formed, GC-MS analysis was performed on a diethyl ether extract after esterification by diazomethane on samples stored 0,60 ,

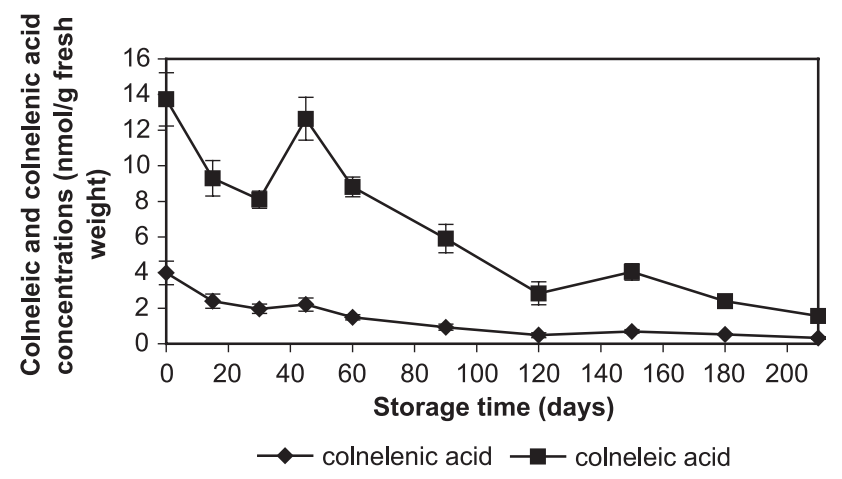

Fig. 2. Colneleic and colnelenic acid concentrations during aging of potato tubers. The acids were extracted with diethyl ether and analysed by HPLC. The analysis was performed on three independent extracts. and 210 days. This technique confirmed the presence of HPOD and HPOT and colneleic acid, and identified 9-oxononanoic acid. HPOD and HPOT identification of individual species was based on retention time compared with pure HPOs esterified with diazomethane. GC-MS was used only for confirmation of the presence of HPOs and not for quantitation (which was preformed by HPLC), because HPOs can undergo thermal rearrangements during GC analysis. Colneleic acid methyl ester was easily identified on the basis of its retention time (40.5 $\mathrm{min})$ and fragmentation pattern $(\mathrm{m} / \mathrm{z}=308(17), 251(5), 165(12), 151(12), 137$ (23), 123 (27), 109 (29), 95 (60), 81 (94), 67 (100), 55 (63)) in comparison with pure compound esterified with diazomethane. 9-oxo-nonanoic acid methyl ester was identified in each of the three extracts $(0,60$ and 210 days of storage) on the basis of its retention time (23 $\mathrm{min})$ and mass spectrum $(\mathrm{m} /$ $z=186$ (2), 143 (4), 127 (9), 87 (40), 74 (100), 59 (22), 55 (35)). Because the colneleic acid and 9-oxo nonanoic acid fragments were identified only in the potato samples and not when pure HPOs were analyzed under the same conditions, it is clear that these compounds originated from the tubers. For highest sensitivity, SIM was employed to search for other oxylipins potentially formed in the extracts. Three ions characteristic of the methyl ester of each oxylipin were monitored (molecular ion and two intense and typical fragments). Detection of the following compounds was attempted: methyl esters of ketodienoic acid, methyl ester of 12-oxo-9Z-dodecenoic acid, methyl ester of 12-oxo-10E dodecenoic acid, methyl ester of epoxyhydroxyoctadecenoic, and decadienoic acid, methyl ester of trihydroxioctadecenoic acid, $\alpha$ and $\gamma$ ketols, methyl ester of jasmonic acid and of oxo-phytodienoic acid. Under our extraction and analysis conditions, none of these species were detected. Analysis of the trimethylsilyl ether derivatives also did not result in detection of other oxylipins (data not shown).

It has previously been shown that colneleic acid is the main oxylipin in potato cells but hydroxy fatty acids and trihydroxy fatty acids were also identified upon elicitor treatment [14]. Colneleic acid is clearly involved in plant defence against pathogens $[6,14]$ and is also an inhibitor of LOX activity [33], but this work is the first indication for a role of colneleic acid during the normal life cycle of the tuber, and for a potential role of colneleic acid in sprout development. It is unlikely that the 9-oxo-nonanoic acid methyl ester in the extracts was formed by the action of HPO lyase on 9-HPOD and HPOT, because it has been demonstrated that potato tuber HPO lyase is specific for 13HPOD and HPOT [30,34], and the tubers contained little 13-HPOD and HPOT. Moreover, HPO lyase activity rapidly drops to zero during tuber storage [30]. Since 9-oxononanoic acid is present even after 210 days of aging, we postulate that it is not formed by HPO lyase, but that it is a degradation product originating from colneleic acid. It has been demonstrated that the degradation pathway of colneleic and colnelenic acid forms the same products as HPO lyase does [35]. 


\subsection{Phospholipid and galactolipid content of potato tubers during aging}

In order to investigate the origin of the fatty acids that are substrates for the lipoxygenase pathway, analysis of phospholipids and galactolipids, was undertaken by ESI-MS/ MS, a rapid and sensitive method for quantitative determination of membrane lipids and their individual molecular species. Lipid profiling by ESI-MS/MS requires only simple sample preparation and small amounts of plant material. This technique has been used previously to profile membrane lipids in a plant stress response [25], and here it is utilized in a new application. Analysis of lipid extracts by ESI-MS/MS allowed the identification and quantification of the principal phospholipids and galactolipids during the storage of potato tubers. Fig. 3 shows the concentrations (nmol/g fresh weight) of the main classes of phospholipids and galactolipids during the storage period. Levels of the major non-plastidic phospholipids, phosphatidylcholine (PC), phosphatidylethanolamine (PE), and phosphatidylinositol (PI), decreased rapidly between 0 and 45 days of storage and remained low at 60 days. Between 60 days and 90 days, when multiple sprouts were growing, the levels of PC, PE, and PI increased 4-5-fold, then remained relatively steady from 90 to 210 days. The major plastidic galactolipids, monogalactosyldiacylglycerol (MGDG) and digalactosyldiacylglycerol (DGDG) followed a similar pattern. Levels of the major plastidic phospholipid, phosphatidylglycerol (PG), underwent much smaller changes with only a small increase toward the end of the storage period. Dehydration (Section 3.1) of the tuber during storage at 20
${ }^{\circ} \mathrm{C}$ accounted for just a small fraction of the observed increases in lipid content between 60 and 90 days, and certainly did not account for the drop in lipid content between 0 and 45 days. The drop in lipid content between 0 and 45 days is likely to be directly related to the large increases in 9-HPOD, 9-HPOT, colneleic acid, and colnelenic acids, whose levels peaked at 45-60 days. Indeed, the amount of these compounds present at 45-60 days was about $100 \mathrm{nmol} / \mathrm{g}$ fresh weight (Figs. 1 and 2). This correlates well with the amount of loss of complex polar lipids between 0 and 45 days, when the total of these species dropped $113 \mathrm{nmol} / \mathrm{g}$ fresh weight; most of the hydrolysed lipids were diacyl species containing about 47\% 18:2 and $15 \% 18: 3$ [24]. On the other hand, the physiological basis for the increases in PC, PE, PI, PA, MGDG, and DGDG levels between 60 and 90 days is less clear, and future investigation of the basis for this increase is warranted.

The relative amounts of the lipid classes also varied during aging. In decreasing prevalence, the main classes of lipids are $\mathrm{PC}>\mathrm{DGDG}>\mathrm{PI}$ at 0 days of storage, while PC $>$ PE $>$ PI after 210 days of storage. These alterations in the relative amounts of $\mathrm{PC}, \mathrm{PE}$, and DGDG are in agreement with those observed during 38-month storage of the potato variety Désirée at $4{ }^{\circ} \mathrm{C}$ by Zabrouskov and Knowles [36], who found that PC and DGDG were the main lipid classes in young tubers while PC and PE were dominant in older samples (38 months). These workers also observed increases in PC and PE levels during storage [36].

Phosphatidic acid (PA), lysophosphatidylcholine (lysoPC), lysophosphatidylethanolamine (lysoPE), and lysophosphatidylglycerol (lysoPG) may be formed by degrada-

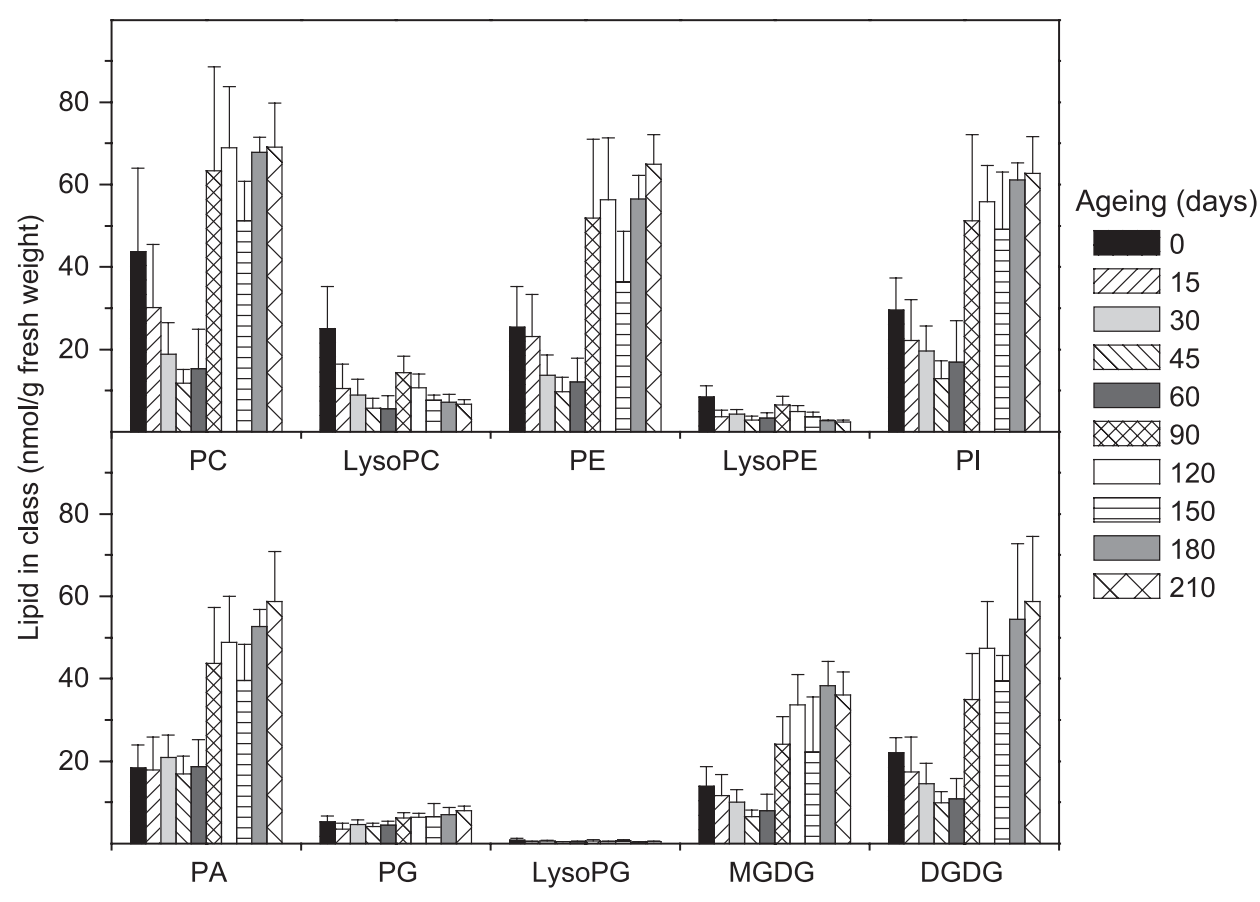

Fig. 3. Phospholipid and galactolipid levels during aging of potato tubers. The analysis was performed by ESI-MS/MS on five independent extracts at each time point. 
tion of the other phospholipid classes. These lipids were present at high levels in potato tuber. Initially, PA accounted for $9.1 \pm 1.1 \mathrm{~mol} \%$ of the total lipid, while lysoPC, lysoPE, and lysoPG accounted for $12.2 \pm 2.5 \%, 4.1 \pm 0.3 \%$, and $0.4 \pm 0.1 \%$ of the total lipids, respectively. In contrast to the other diacyl phospholipids, except PG, PA levels remained constant for the initial 60 days of storage. Interestingly, lysoPC and lysoPE levels dropped during this period, like their diacyl counterparts. These data suggest that the lipase(s) that produce the free fatty acids used as LOX substrates must have removed the fatty acids from both the $s n 1$ and $s n 2$ positions of polar lipids, without accumulation of lysoPC or lysoPE. In fact, lipases found in potato tuber vacuoles, the patatins, have low specificity; recently it was demonstrated that a recombinant patatin-like Arabidopsis enzyme is capable of hydrolysing acyl groups from both $s n 1$ and $s n 2$ positions of phospholipids [37]. Between 60 and 90 days of aging, PA, lysoPC, and lysoPE levels all increased $2-3$-fold. LysoPC and lysoPE levels dropped again between 90 and 210 days of storage. These changes resulted in a continuous drop throughout the aging period in the mole percentage of lysoPC and lysoPE in the total lipids, so that at 210 days, lysoPC accounted for only $1.9 \pm 0.2 \%$ and lysoPE for $0.7 \pm 0.1 \%$ of the total lipid.
The molecular species of membrane lipids at 0,60 , and 210 days are shown in Fig. 4. ESI-MS/MS analysis allows us to determine the amounts of individual lipid molecular species. In each head group class, species are identified in terms of the total number of acyl carbons and the total number of double bonds. Molecular species containing 34 carbons and two double bonds are major molecular species of PC, PE, PI, PA, PG, and DGDG, while molecular species containing 36 carbons and four double bonds are prominent species of PC, PE, PA, and DGDG. Since potato tubers contain high levels (47\%) of 18:2 and very low levels $(0.5 \%)$ of $18: 1$ [24], the $34: 2$ species must be largely a combination of $16: 0$ and 18:2 (rather than 18:1-18:1), while the $36: 4$ species must be largely an 18:2-18:2 combination (rather than 18:1-18:3). The most abundant lysophospholipid acyl species was 18:2. Examination of Fig. 4 shows that the ratio of the $36: 4$ to $34: 2$ species of PC, PE, PI, and PA tended to be lower at 60 days than at either 0 or 210 days. This suggests that there may have been some preferential hydrolysis of $36: 4$ (18:2-18:2) as compared to other molecular species of PC, PE, PI, and PA at the time of formation of 9-HPOD and colneleic acid, and that perhaps $36: 4(18: 2-18: 2)$ species were preferentially hydrolysed, releasing the LOX substrate, 18:2. Similarly, the 18:2

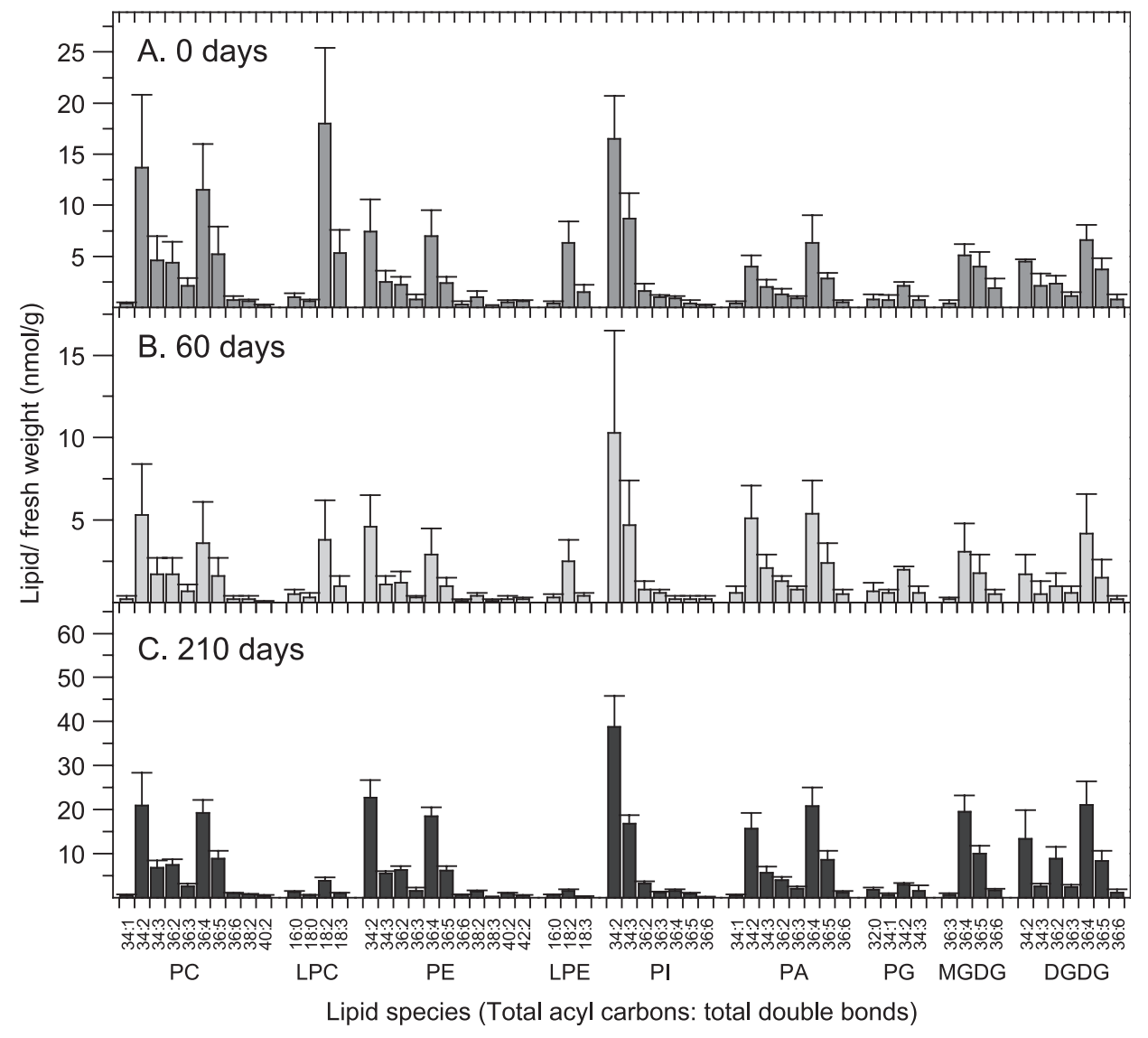

Fig. 4. Phospholipid and galactolipid molecular species during aging of potato tubers. Note the differences in the scales among the three graphs. Species with masses significantly greater than $0.2 \mathrm{nmol} / \mathrm{g}$ fresh weight are shown. (A) Aging for 0 days. (B) Aging for 60 days. (C) Aging for 210 days. The analysis was performed by ESI-MS/MS on five independent extracts at each time point. 
species of lysoPC and lysoPE dropped to a greater extent than the other lyso molecular species between 0 and 60 days, suggesting that these 18:2 lysophospholipids might also have undergone some preferential hydrolysis. However, essentially all molecular species of PC, PE, PI, MGDG, and DGDG were decreased at 60 days compared to 0 days (Figs. 3 and 4; note scales in Fig. 4), and any discrimination among molecular species by the enzymes that catalysed the lipid degradation that occurred between 0 and 60 days was a subtle phenomenon.

In a previous study, we determined that membrane integrity dropped drastically between 120 and 240 days of aging [24]. In that study, we showed that that the double bond index (DBI) of fatty acid species of potato tubers fluctuated during aging of tubers. ESI-MS/MS analysis of phospholipids and galactolipids confirmed that there were no major shifts toward either more unsaturated or saturated molecular species (Fig. 4). Thus, it is unlikely that changes in unsaturation contributed significantly to the alterations in membrane integrity that were observed previously. Since levels of fatty acid HPOs were also relatively low at the end of the storage period, it is also unlikely that the presence of these species contributed to the loss of membrane integrity. Our ESI-MS/MS data demonstrate that incorporation into complex lipids was not a major fate of hydroperoxy fatty acids. No increases were detected during aging in complex lipid species with masses that might be expected for species containing hydroperoxides. For example, 18:2-HPOD PC, would have the same nominal mass as $38: 2 \mathrm{PC}$, and the species that we have identified as " $38: 2$ PC" could potentially include some 18:2-HPOD PC. However, this species was present in low amounts ( $1.4 \mathrm{~mol} \%$ of PC species), and there was no increase in the mole percentage of the species identified as "38:2 PC" during aging (Fig. 4). LysoPCs and lysoPEs, which can destabilize membrane structure, were also at their lowest levels at the end of the storage period. On the other hand, the mole percentages of PE and PA increased from $12 \%$ and $9 \%$ of the total polar lipids to $18 \%$ and $16 \%$, respectively, while PC levels dropped slightly, from $21 \%$ to $19 \%$ during the aging. These changes might have led to formation of non-bilayer lipids that destabilized membrane structure, since both PE and PA have the capacity to form non-bilayer phases $[38,39]$.

\section{Concluding remarks}

Our results demonstrate that, during aging and sprouting in potato tubers, the LOX pathway is activated. The LOX pathway seems to be finely regulated as high enzymatic activities and product accumulation are restricted to a narrow window of time during the storage period, with levels of products peaking at 45-60 days of aging. Galactolipases and phospholipases liberate free fatty acids, including 18:2, which are peroxidised to HPOs by LOX. HPOs are then converted by DES to colneleic acid, which is degraded to 9-oxo-nonanoic acid. The roles of colneleic acid and 9-oxo-nonanoic acid in tubers during aging remain to be determined. We have previously demonstrated that membrane integrity drops after 120 days of tuber aging. However, during that late period of aging, fatty acid HPO content is low. Thus, oxygenated lipid species are not the main cause of the loss of membrane integrity.

\section{Acknowledgements}

Marie-Laure Fauconnier is a postdoctoral researcher of the Fonds National pour la Recherche Scientifique of Belgium. The authors thank Jean-Luc Montillet (Cadarache, France) for the determination of R/S ratio in HPO and for the analysis of 12- and 16-HPOT. The authors thank Catherine Collin for her efficient help and rigorous work. We thank Dr. Todd Williams of the University of Kansas Mass Spectrometry Laboratory for collecting the ESI-MS/ MS data. We also want to acknowledge the National Science Foundation (USA) for supporting the development of lipid profiling (NSF MCB-0110979).

\section{References}

[1] E. Blée, Impact of phyto-oxylipins in plant defense, Trends Plant Sci. 7 (2002) 315-322.

[2] I. Feussner, C. Wasternack, The lipoxygenase pathway, Annu. Rev. Plant Biol. 53 (2002) 275-297.

[3] A.N. Grechkin, Hydroperoxide lyase and divinyl ether synthase, Prostaglandins Other Lipid Mediat. 68-69 (2002) 457-470.

[4] T. Galliard, D.R. Phillips, The enzymic conversion of linoleic acid into 9-(Nona-1', $3^{\prime}$-dienoxy)non-8-enoic acid, a novel unsaturated ether derivative isolated from homogenates of Solanum tuberosum tubers, Biochem. J. 129 (1972) 743-753.

[5] D. Caldelari, E.E. Farmer, A rapid assay for the coupled cell free generation of oxylipins, Phytochemistry 47 (1998) 599-604

[6] H. Weber, A. Chételat, D. Caldelari, E.E. Farmer, Divinyl ether fatty acid synthesis in late-blight-diseased potato leaves, Plant Cell 11 (1999) 485-493.

[7] A.N. Grechkin, F.N. Fazliev, L.S. Mukhatarova, The lipoxygenase pathway in garlic (Allium sativum L.) bulbs: detection of the novel divinyl ether oxylipins, FEBS Lett. 371 (1995) 159-162.

[8] M. Hamberg, A pathway for biosynthesis of divinyl ether fatty acids in green leaves, Lipids 33 (1998) 1061-1071.

[9] M. Hamberg, Biosynthesis of new divinyl ether oxilipins in Ranunculus plants, Lipids 37 (2002) 427-433

[10] P.J. Proteau, W.H. Gerwick, Divinyl ethers and hydroxy fatty acids from three species of Laminaria, Lipids 28 (1993) 783-787.

[11] Z.D. Jiang, W.H. Gerwick, Novel oxilpins from the temperate red algae Polyneura latissima: evidence for an arachidonate $9(S)$ lipoxygenase, Lipids 32 (1997) 231-235.

[12] E. Blée, Phytooxylipins and plant defense reactions, Prog. Lipid Res. 37 (1998) 33-72.

[13] H. Weber, Fatty acids derived signals in plants, Trends Plant Sci. 7 (2002) $217-224$.

[14] C. Göbel, I. Feussner, A. Schmidt, D. Schell, J. Sanchez-Serrano, M. Hamberg, S. Rosahl, Oxylipin profiling reveals the preferential stimulation of the 9-lipoxygenase pathway in elicitor-treated potato cells, J. Biol. Chem. 276 (2001) 6267-6273.

[15] M. Stumpe, R. Kandzia, C. Göbel, S. Rosahl, I. Feussner, A pathogen 
inducible divinyl ether synthase (CYP 74D) from elicitor treated potato suspension cells, FEBS Lett. 507 (2001) 371-376.

[16] C. Göbel, I. Feussner, M. Hamberg, S. Rosahl, Oxylipin profiling in pathogen infected potato leaves, Biochim. Biophys. Acta 1584 (2002) $55-64$.

[17] M. Fukuchi-Mizutani, K. Ishiguro, T. Nakayam, Y. Utsunomiya, Y. Tanaka, T. Kusumi, T. Ueda, Molecular and functional characterization of a rose lipoxygenase cDNA related to flower senescence, Plant Sci. 160 (2000) 129-137.

[18] T. Panavas, B. Rubinstein, Oxidative events during programmed cell death in daylily (Hemerocallis hybrid) petals, Plant Sci. 133 (1998) $125-138$

[19] M.A. Rouet-Mayer, J.M. Bureau, C. Laurière, Identification and characterization of lipoxygenase isoforms in senescing carnation petals, Plant Physiol. 98 (1992) 971-978.

[20] S. Grossman, Y.Y. Leshem, Lowering of endogenous lipoxygenase activity in Pisum sativum foliage by cytokinin as related to senescence, Plant Physiol. 43 (1978) 359-362.

[21] J.M. Sung, C.C. Chiu, Lipid peroxide-scavenging enzymes of naturally aged soybean seed, Plant Sci. 110 (1995) 45-52.

[22] T.K. Peterman, J.N. Siedow, Behavior of lipoxygenase during establishment, senescence, rejuvenation of soybean cotyledons, Plant Physiol. 78 (1985) 690-695.

[23] M. Kar, J. Feierabend, Metabolism of activated oxygen in detached wheat and rye leaves and its relevance to the initiation of senescence, Planta 160 (1984) 385-391.

[24] M.L. Fauconnier, J. Rojas-Beltran, J. Delcarte, F. Dejaeghere, M. Marlier, P. du Jardin, Lipoxygenase pathway and membrane permeability and composition during storage of potato tuber (Solanum tuberosum L. cv Bintje and Désirée) in different conditions, Plant Biol. 4 (2002) 77-85.

[25] R. Welti, W. Li, M. Li, Y. Sang, H. Biesiada, H.E. Zhou, C.B. Rajashekar, T.D. Williams, X. Wang, Profiling membrane lipids in plant stress responses, J. Biol. Chem. 277 (2002) 31994-32002.

[26] M.L. Fauconnier, A.G. Perez, C. Sanz, M. Marlier, Purification and characterisation of tomato leaf (Lycopersicon esculentum Mill.) hydroperoxide lyase, J. Agric. Food Chem. 45 (1997) 4232-4236.

[27] N. Degousée, C. Triantaphylides, J.L. Montillet, Involvement of oxidative processes in the signaling mechanisms leading to the activation of glyceollin synthesis in soybean (Glycine max), Plant Physiol. 104 (1994) 945-952.

[28] C. Rusterucci, J.L. Montillet, J.P. Agnel, C. Battesti, B. Alonso, A.
Knoll, J.J. Bessoule, P. Etienne, L. Suty, J.P. Blein, C. Triantaphylades, Involvement of lipoxygenase-dependent production of fatty acid hydroperoxides in the development of the hypersensitive cell death induced by cryotogein on tobacco leaves, J. Biol. Chem. 274 (2002) 36446-36455.

[29] P. Ellishèche, Aspects physiologiques de la croissance et du developpement, in: P. Rouselle, Y. Robert, J.C. Crosnier (Eds.), La pomme de terre, INRA, Paris, 1996, pp. 72-124.

[30] M.L. Fauconnier, J. Delcarte, M. Jaziri, P. du Jardin, M. Marlier, Fatty acid hydroperoxide biotransformation by potato tuber cell-free extracts, J. Plant Physiol. 159 (2002) 1055-1060.

[31] S. Berger, H. Weichert, A. Porzel, C. Wasternack, H. Kühn, I. Feussner, Enzymatic and non-enzymatic lipid peroxidation in leaf development, Biochim. Biophys. Acta. 55821 (2001) 1-11.

[32] E. Mulliez, J.P. Leblanc, J.J. Girerd, M. Rigaud, J.C. Chottard, 5Lipoxygenase from potato tuber. Improved purification and physiological characteristics, Biochim. Biophys. Acta 916 (1987) 13-23.

[33] E. Corey, R. Nagata, S. Wright, Biomimetic total synthesis of colneleic acid and its function as lipoxygenase inhibitor, Tetrahedron Lett. 28 (1987) 4917-4920.

[34] G. Vancanneyt, C. Sanz, T. Farmaki, M. Paneque, F. Ortego, P. Castanera, J. Sanchez-Serrano, Hydroperoxide lyase depletion in transgenic potato plants leads to an increase in aphid performance, Plant Biol. 98 (2001) 8139-8144.

[35] T. Galliard, D.A. Wardale, J.A. Matthew, The enzymic and non-enzymic degradation of colneleic acid an unsaturated fatty acid ether intermediate in the lipoxygenase pathway of linoleic acid oxidation in potato (Solanum tuberosum) tubers, Biochem. J. 138 (1974) 23-31.

[36] V. Zabrouskov, N.R. Knowles, Changes in lipid molecular species and sterols of microsomal membranes during aging of potato (Solanum tuberosum L.) seed-tubers, Lipids 37 (2002) 309-315.

[37] A. Holk, S. Rietz, M. Zahn, H. Quader, G.F.E. Scherer, Molecular identification of cytosolic, patatin-related phospholipases A from Arabidopsis with potential functions in signal transduction, Plant Physiol. 130 (2002) 90-101.

[38] A.J. Verleij, R. DeMaagd, J. Leunissen-Bijvelt, B. DeKruijff, Divalent cations and chlorpromazine can induce non-bilayer structures in phosphatidic acid-containing membranes, Biochim. Biophys. Acta. 684 (1982) 255-262.

[39] P.R. Cullis, B. DeKruijff, Lipid polymorphism and the functional roles of lipids in biological membranes, Biochim. Biophys. Acta 559 (1979) 399-420. 